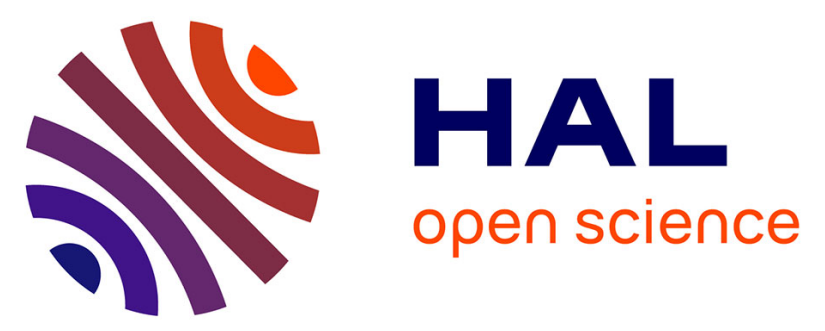

\title{
Scattering Centers Monitoring in Refocused SAR Images on a High Resolution DEM
}

Andrei Anghel, Gabriel Vasile, Cornel Ioana, Remus Cacoveanu, Silviu Ciochina, Jean-Philippe Ovarlez, Rémy Boudon, Guy d’Urso, Irena Hajnsek

\section{- To cite this version:}

Andrei Anghel, Gabriel Vasile, Cornel Ioana, Remus Cacoveanu, Silviu Ciochina, et al.. Scattering Centers Monitoring in Refocused SAR Images on a High Resolution DEM. IGARSS 2014 - IEEE International Geoscience and Remote Sensing Symposium, Jul 2014, Québec, Canada. pp.1883-1886, 10.1109/IGARSS.2014.6946824 . hal-01104814

\section{HAL Id: hal-01104814 \\ https://hal-centralesupelec.archives-ouvertes.fr/hal-01104814}

Submitted on 4 Jun 2021

HAL is a multi-disciplinary open access archive for the deposit and dissemination of scientific research documents, whether they are published or not. The documents may come from teaching and research institutions in France or abroad, or from public or private research centers.
L'archive ouverte pluridisciplinaire HAL, est destinée au dépôt et à la diffusion de documents scientifiques de niveau recherche, publiés ou non, émanant des établissements d'enseignement et de recherche français ou étrangers, des laboratoires publics ou privés. 


\title{
SCATTERING CENTERS MONITORING IN REFOCUSED SAR IMAGES ON A HIGH-RESOLUTION DEM
}

\author{
Andrei Anghel ${ }^{\star \dagger} \quad$ Gabriel Vasile ${ }^{\dagger} \quad$ Cornel Ioana $^{\dagger} \quad$ Remus Cacoveanu ${ }^{\star} \quad$ Silviu Ciochina ${ }^{\star}$ \\ Jean-Philippe Ovarlez ${ }^{\ddagger}$ Rémy Boudon ${ }^{\S}$ Guy d'Urso ${ }^{\S}$ Irena Hajnsek \\ ${ }^{\star}$ University POLITEHNICA of Bucharest, Faculty of Electronics, Telecommunications and \\ Information Technology, Telecommunications Department, Bucharest, Romania \\ ${ }^{\dagger}$ CNRS/Grenoble INP, Grenoble-Image-sPeach-Signal-Automatics Lab, Grenoble, France \\ $\ddagger$ DEMR/TSI, ONERA, Palaiseau, France \\ $\S$ Électricité de France, EDF DTG / R\&D, France \\ ฯ Microwaves and Radar Institute, German Aerospace Center (DLR), Wessling, Germany
}

\begin{abstract}
Infrastructure monitoring applications can require the tracking of slowly moving points of a certain structure. Given a certain point from a structure to be monitored, in the context of available SAR products where the image is already focused in a slant range - azimuth grid, it is not obvious if this point is the scattering center, if it is in layover or if it is visible from the respective orbit. This paper proposes a scattering center monitoring procedure based on refocusing a set of SAR images on a provided high-resolution DEM of the structure. The scattering centers of the refocused image are detected in the 4-D tomography framework by testing if the main response is at zero elevation in the local elevation-velocity spectral distribution obtained using the Capon estimator. The algorithm is validated on real data acquired with the TerraSAR-X satellite over the Puylaurent water dam in France during March-June 2012. The relative displacements between scattering regions show very good agreement with the in situ measurements.
\end{abstract}

Index Terms - Synthetic Aperture Radar (SAR), Refocusing, Tomography, Interferometry.

\section{INTRODUCTION}

In infrastructure monitoring, it can be often necessary to track the slow displacements of certain points of a given structure (building, water dam, landslide, etc.). This can be done using current spaceborne civil sensors, such as TerraSAR-X and TanDEM-X provided their short wavelength of $3.1 \mathrm{~cm}$, the short revisit time of 11 days and especially the $1 \mathrm{~m}$ resolution in spotlight mode [1,2]. Still, given a certain infrastructure element, from one acquisition geometry only one side of the structure can be observed. Due to typical side-look effects (layover ambiguities, multi-path scattering effects or shadowing) it is not always clear which points are actual scattering centers and consequently which of them can be accurately monitored. If the coordinates of a number of points from the structure are known with at least centimeter accuracy (measured with GPS or LIDAR techniques) their response (if there is any) could be determined if the raw data were focused on a 3-D grid containing precisely these points. However, in most cases in the delivered products the SAR images are already focused on a slant range-azimuth grid which is not related to any specific scatterer.

Since the availability and processing of spaceborne raw SAR data is not very convenient, this paper proposes a scattering center monitoring method based on refocusing the SAR data on a given DEM containing the points of the structure that needs to be monitored. The real scatterers which provide the main response and are not faded by layover are detected in the context of differential tomography $[3,4,5]$. This is obtained by exploiting the fact that each refocused scattering center will be at zero elevation in the local elevation-velocity (EV) plane.

An advantage of the refocusing approach on a specific grid is that no shifting or resampling (as part of the coregistration process) are needed because the samples get automatically aligned by refocusing each image on the same grid. Furthermore, in comparison with the classical coregistered inteferograms, a highly reflective scatterer with known coordinates that needs to be tracked (a mounted corner reflector for instance) cannot have an inconvenient off-grid position for processing in the SAR image (e.g.: to be at the edge of 4 neighboring pixels), it will always be placed in the center of the resolution cell on the new grid and its relative displacements will be accurately measured.

The monitoring technique is investigated on a set of images acquired by the TerraSAR-X satellite over the Puylaurent water dam in France between March-June 2012.

The remaining of the paper is organized as follows. Section II presents the scattering center monitoring algorithm and is divided in two parts. The refocusing procedure is discussed first. Then, the detection and tracking based on the 4-D tomography framework are described. Section III shows the 
results of the monitoring procedure applied to TerraSAR-X data. Finally, the conclusions are stated in Section IV.

\section{SCATTERING CENTERS MONITORING PROCEDURE}

The scattering centers monitoring procedure consists of two steps: the refocusing of the acquired SAR images on the provided DEM and the real scattering centers detection along with the displacements computation.

\subsection{Refocusing on a given DEM}

In order to focus the data on a certain DEM, the first step is to defocus the image in azimuth which is mainly a reversed version of the SPECAN processing used for azimuth focusing $[6,7]$. This approach is possible because the azimuth scaling consists only of Fourier transforms and complex multiplications which are reversible. The first operation is to select from the initial image the slant range-azimuth region containing the target. Because the range compression is not modified during the processing, the selected region can be cropped in range in order to reduce the computation time. In the case of spotlight SAR images the azimuth sampling frequency is larger then the raw data pulse repetition frequency (PRF) in order to cover the complete spotlight bandwidth. In the defocusing procedure the sampling frequency is the one of the SAR image, so the subaperture approach used for real-time focusing [8] doesn't need to be employed. A detailed description of the azimuth defocusing procedure was given in [9]. After reversing the azimuth focusing steps from [7], the defocused signal for $N$ scatterers in the slant range-azimuth time domain has the form:

$$
\begin{aligned}
& s_{d}\left(t, r_{0}\right)=\sum_{i=1}^{N} A_{i} \operatorname{sinc}\left[\frac{2 \pi B_{r}}{c}\left(r_{0}-r_{0, i}\right)\right] \\
& \times \operatorname{rect}\left[\frac{t-t_{i}}{t_{A P}}\right] \exp \left(-j \frac{4 \pi}{\lambda} \sqrt{r_{0}^{2}+\left[v_{0}\left(t-t_{i}\right)\right]^{2}}\right),
\end{aligned}
$$

where $t$ is the azimuth (slow) time axis, $r_{0}$ is the closest approach slant range axis, $B_{r}$ is the range chirp bandwidth, $v_{0}$ is the sensor's zero Doppler velocity, $c$ is the speed of light and $t_{A P}$ is the equivalent synthetic aperture (illumination) time [1]. $A_{i}, t_{i}, r_{0, i}$ represent respectively the complex amplitude, the zero Doppler azimuth time and the slant range at closest approach for the scatterer $i$. Notice that the signal in (1) has the natural hyperbolic phase history for each target and is not affected by range migration. Seen as a matrix $s_{d}[m, n]=s_{d}(m \delta t, n \delta r)$, the phase history for a certain closest approach slant range is placed on one column and each line contains a range profile.

The grid focusing procedure starts by extracting the annotated orbit data. The envisaged geometry is shown in Fig. 1(a). The unit vector $\overrightarrow{\boldsymbol{u}}$ of the azimuth direction is computed as the normalized velocity vector of the satellite at the azimuth time of the image center. For an azimuth resolution larger than $1 \mathrm{~m}$ the straight line trajectory approximation is satisfactory (the curved orbit correction is needed only for staring spotlight mode [10]). The position of the satellite's antenna phase center (APC) at a given azimuth time $t$ can be written as:

$$
\overrightarrow{\boldsymbol{r}}_{\boldsymbol{a}}(t)=\overrightarrow{\boldsymbol{r}}_{\boldsymbol{a}, \mathbf{0}}+v_{0} t \overrightarrow{\boldsymbol{u}},
$$

where $\overrightarrow{\boldsymbol{r}}_{\boldsymbol{a}, \mathbf{0}}$ is the APC position vector at $t=0$. For a given target having the position vector $\overrightarrow{\boldsymbol{r}}_{\boldsymbol{k}}$, the closest approach distance to the synthetic aperture is given by

$$
r_{0, k}=\left\|\left(\overrightarrow{\boldsymbol{r}}_{a, 0}-\overrightarrow{\boldsymbol{r}}_{\boldsymbol{k}}\right)-\left[\left(\overrightarrow{\boldsymbol{r}}_{a, 0}-\overrightarrow{\boldsymbol{r}}_{\boldsymbol{k}}\right) \cdot \overrightarrow{\boldsymbol{u}}\right] \overrightarrow{\boldsymbol{u}}\right\|
$$

and its response is computed as

$$
g\left(\overrightarrow{\boldsymbol{r}}_{\boldsymbol{k}}\right)=\sum_{m=M_{1}}^{M_{2}} s_{d}\left(m \delta t, r_{0, k}\right) \exp \left(j \frac{4 \pi f_{c}}{c}\left\|\overrightarrow{\boldsymbol{r}}_{\boldsymbol{a}}(m \delta t)-\overrightarrow{\boldsymbol{r}}_{\boldsymbol{k}}\right\|\right),
$$

where $M_{1}$ and $M_{2}$ are the limits corresponding to the illumination duration of the respective target. The refocusing is implemented by applying (4) to each point of the given DEM.

\subsection{Detection and Tracking of Scattering Centers}

For two refocused images, the stable scatterers could be detected by classical coherence evaluation on a vicinity of each refocused point. However, in order to determine if the reflecting scattering center is actually at the given point an approach based on a series of acquisitions is needed in order to create an elevation aperture. Considering the 4-D SAR imaging model in [3] for each scatterer $k$ from the given DEM (on-grid target situated at $\vec{r}_{\boldsymbol{k}}$ ) the received signal vector is written as:

$$
\boldsymbol{g}\left(\overrightarrow{\boldsymbol{r}}_{\boldsymbol{k}}\right)=\int_{\Delta s} \int_{\Delta v} p_{\gamma}(s, v) \mathbf{a}(s, v) d s d v
$$

where $p_{\gamma}(s, v)=\gamma(s) p(s, v)$, with $\gamma(s)$ being the reflectivity profile along elevation $s$ and $p(s, v)$ the elevation-velocity spectral distribution of the displacement terms. $\Delta s$ and $\Delta v$ are the elevation and velocity supports of $p_{\gamma}(s, v)$ and $\mathbf{a}(s, v)$ is the steering vector whose elements are defined as:

$$
a_{n}(s, v)=\exp \left[j 2 \pi\left(\frac{2 b_{\perp, n}}{\lambda r_{0, k}} s+\frac{2 t_{n}}{\lambda} v\right)\right] .
$$

In (6), $b_{\perp, n}$ is the orthogonal baseline computed for the target $k$ (perpendicular to $\overrightarrow{\boldsymbol{r}}_{\mathbf{0}, \boldsymbol{k}}$ ) relative to the first acquisition, $t_{n}$ is the acquisition time and $\lambda$ is the central wavelength. Because the $\left(b_{\perp, n}, t_{n}\right)$ pairs are sparse and nonuniform, the function $p_{\gamma}(s, v)$ is reconstructed using the Capon filter $[5,11]$ :

$$
\hat{p_{\gamma}}(s, v)=\frac{\mathbf{a}^{H}(s, v) \hat{\mathbf{R}}^{-1} \boldsymbol{g}\left(\overrightarrow{\boldsymbol{r}}_{\boldsymbol{k}}\right)}{\mathbf{a}^{H}(s, v) \hat{\mathbf{R}}^{-1} \mathbf{a}(s, v)},
$$

where $\hat{\mathbf{R}}$ is a multi-look estimate of the data vector $\boldsymbol{g}\left(\overrightarrow{\boldsymbol{r}}_{\boldsymbol{k}}\right)$ covariance matrix. The power spectral density (PSD) is then 


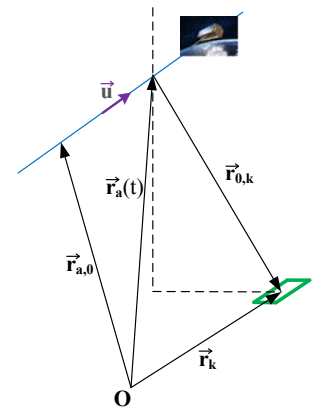

(a)

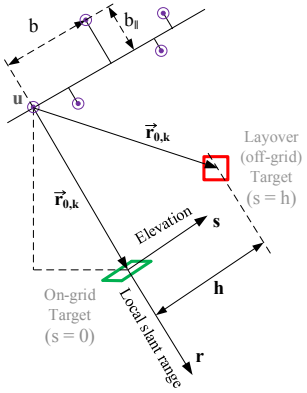

(b)
Fig. 1. (a) Refocusing geometry for a target with a known on-grid position $\overrightarrow{\boldsymbol{r}}_{\boldsymbol{k}}$. (b) Layover detection geometry based on zero elevation for on-grid targets.

obtained as the power of $\hat{p_{\gamma}}(s, v)$. By changing the geometrical $\mathrm{EV}$ configuration for each refocused target, its position on the elevation axis is at $s=0$ and any other scatterer situated in layover will be at another distance as presented in Fig. 1(b). Therefore the persistent scatterers from the set of targets which have the real scattering center at the given position and are not affected by layover are the ones that have only one significant maximum value of the PSD in the EV plane at zero elevation. The mean displacement velocity (MDV) $\bar{v}$ of a detected scatterer is estimated as the location of the peak on the velocity axis at zero elevation.

The displacements time series $d\left(t_{n}\right)$ of a scatterer can be viewed as a sum between the linear displacement and the residual un-modeled motion. Hence, for a single scatterer placed at zero elevation and unaffected by layover the time series can be written as:

$$
d\left(t_{n}\right)=\bar{v} t_{n}+\frac{\lambda}{4 \pi} \arg \left\{g_{n}\left(\vec{r}_{k}\right) \exp \left(-j \frac{4 \pi \bar{v} t_{n}}{\lambda}\right)\right\} .
$$

This expression is similar to the permanent scatterers interferometry (PSI) case except that here the linear displacement phase term is first subtracted.

\section{RESULTS AND DISCUSSION}

The proposed monitoring algorithm was tested on a set of high-resolution spotlight images acquired with the TerraSARX satellite over the Puylaurent dam in France between MarchJune 2012. Due to the doppler centroid variation of spotlight images a few preprocessing steps (described in [1]) aimed at achieving a common azimuth bandwidth in all images are necessary before defocusing. Also, in order to achieve a centimeter level accuracy the distances computed in (2) and (3) have to be corrected with the annotated atmospheric shifts. A set of 8 images acquired on the descending orbit of the
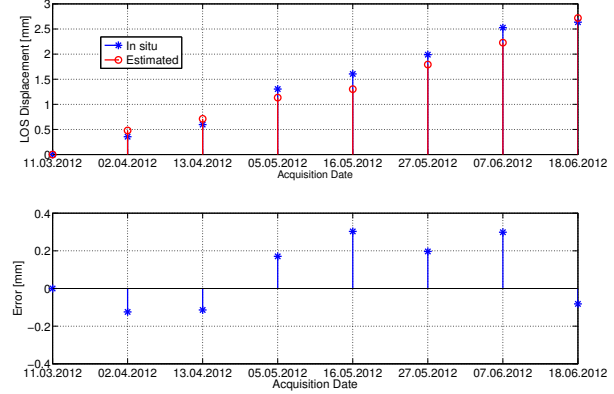

Fig. 3. The Puylaurent dam, TerraSAR-X data, 2012: Estimated vs. in situ LOS displacements and the corresponding errors.

TerraSAR-X is considered in the following analysis. Each image was refocused on a LIDAR measured high-resolution DEM having an average spacing of $0.5 \mathrm{~m}$ between adjacent points. Fig. 2(a) shows the first acquired image (in slant range-azimuth geometry). In Fig. 2(b) is presented the refocused image on the given DEM. Notice that in the 3-D image the highly reflective regions from the initial image appear at different heights and only a part of them are actually provided by the dam's ridge. In order to detect which points from the ridge are scattering centers, the 4-D tomographic detection and tracking procedure was applied on the points from the ridge area. For each refocused point an additional phase difference was employed using as reference a stable point situated at the dam's edge in order to compensate any residual atmospheric effects. The PSD for each point was computed using the Capon estimator. The covariance matrix was estimated on 9 neighboring points. In Fig. 2(c) are plotted the points which have the maximum PSD value at zero elevation and signal to noise ratio of at least $15 d B$ (the noise floor was considered the average level in the water near the dam). The points surrounding the dam's edge have the MDV equal to $0 \mathrm{~mm} / 11$ days (the unit was picked in accordance with the satellite's revisit time) because this area was chosen as reference for the additional phase difference. The displacements time series were computed for points on the dam's ridge where in situ data were available. The obtained relative displacements between two points on the dam's ridge in line of sight (LOS) for each satellite pass are presented in Fig. 3. The first pass was taken as reference and hence appears with zero displacement. The movement projected in the LOS has a linear trend with no significant un-modeled motion and the error is quite small (below $0.5 \mathrm{~mm}$ ).

\section{CONCLUSIONS}

A scattering centers monitoring procedure for refocused SAR images on a given DEM was presented. The efficiency of the proposed method was tested on real data acquired with the TerraSAR-X satellite. The computed relative displacements 


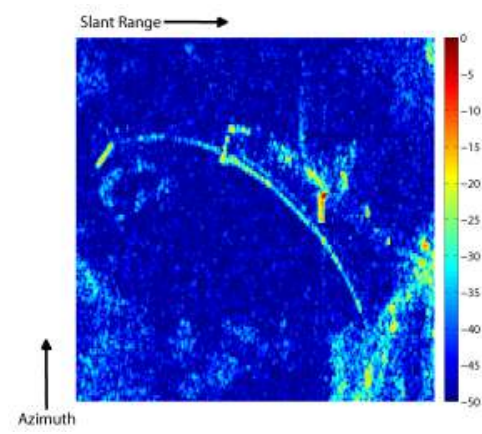

(a)

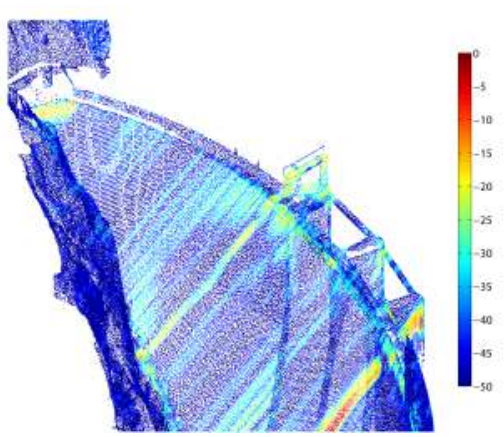

(b)

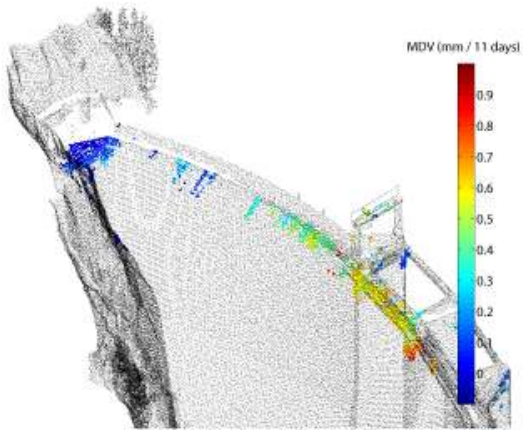

(c)

Fig. 2. The Puylaurent dam. (a) High-Resolution Spotlight TerraSAR-X SAR image acquired on 11 March 2012. (b) Refocused TerraSAR-X image on the LIDAR measured DEM. (c) The detected scattering centers and their MDV superimposed over the provided DEM.

for the detected scattering regions were in very good agreement with the in situ data.

\section{ACKNOWLEDGMENT}

The authors would like to thank the German Aerospace Center (DLR) for providing the TerraSAR-X and TanDEM-X SAR images through the MTH0828 and the NTIINSA2171 projects. Thanks to Dr. Pau Prats (DLR) for the useful discussions regarding the refocusing of high-resolution spotlight SAR images acquired in the TanDEM-X mission. The authors would also like to thank the Électricité de France (EDF) company for providing the in situ measurements for the Puylaurent dam.

\section{REFERENCES}

[1] M. Eineder, N. Adam, R. Bamler, N. Yague-Martinez, and H. Breit, "Spaceborne spotlight sar interferometry with terrasar-x," Geoscience and Remote Sensing, IEEE Transactions on, vol. 47, no. 5, pp. 1524-1535, 2009.

[2] T. Fritz, C. Rossi, N. Yague-Martinez, F. RodriguezGonzalez, M. Lachaise, and H. Breit, "Interferometric processing of tandem-x data," in Geoscience and Remote Sensing Symposium (IGARSS), 2011 IEEE International, 2011, pp. 2428-2431.

[3] F. Lombardini, "Differential tomography: a new framework for sar interferometry," Geoscience and Remote Sensing, IEEE Transactions on, vol. 43, no. 1, pp. 3744, 2005.

[4] G. Fornaro, D. Reale, and F. Serafino, "Fourdimensional sar imaging for height estimation and monitoring of single and double scatterers," Geoscience and Remote Sensing, IEEE Transactions on, vol. 47, no. 1, pp. 224-237, 2009.
[5] F. Lombardini and M. Pardini, "Superresolution differential tomography: Experiments on identification of multiple scatterers in spaceborne sar data," Geoscience and Remote Sensing, IEEE Transactions on, vol. 50, no. 4, pp. 1117-1129, 2012.

[6] A. Moreira, J. Mittermayer, and R. Scheiber, "Extended chirp scaling algorithm for air- and spaceborne sar data processing in stripmap and scansar imaging modes," Geoscience and Remote Sensing, IEEE Transactions on, vol. 34, no. 5, pp. 1123-1136, 1996.

[7] J. Mittermayer, A. Moreira, and O. Loffeld, "Spotlight sar data processing using the frequency scaling algorithm," Geoscience and Remote Sensing, IEEE Transactions on, vol. 37, no. 5, pp. 2198-2214, 1999.

[8] A. Moreira, "Real-time synthetic aperture radar (sar) processing with a new subaperture approach," Geoscience and Remote Sensing, IEEE Transactions on, vol. 30, no. 4, pp. 714-722, 1992.

[9] A. Anghel, G. Vasile, C. Ioana, R. Cacoveanu, S. Ciochina, J.-P. Ovarlez, R. Boudon, and G. d'Urso, "SAR Images Refocusing and Scattering Center Detection for Infrastructure Monitoring," in 2014 IEEE Radar Conference, Cincinnati, Ohio, USA, May 2014, pp. 334-339.

[10] P. Prats-Iraola, R. Scheiber, M. Rodriguez-Cassola, S. Wollstadt, J. Mittermayer, B. Brautigam, M. Schwerdt, A. Reigber, and A. Moreira, "High precision sar focusing of terrasar-x experimental staring spotlight data," in Geoscience and Remote Sensing Symposium (IGARSS), 2012 IEEE International, 2012, pp. 35763579.

[11] J. Capon, "High-resolution frequency-wavenumber spectrum analysis," Proceedings of the IEEE, vol. 57, no. 8, pp. 1408-1418, 1969. 